\title{
Vibration Analysis of The End Conveyor Discharge Arm
}

\author{
G.Y. Lin, W.Z. Wang \\ Department of Mechanical Engineering and Automation \\ Northeastern University \\ Shenyang, Liaoning, China
}

\author{
Z.Y. Li \\ ShanDong Ke Rui Group \\ China
}

\begin{abstract}
End conveyor is widely used in large open-pit mine, in order to gain the three diagonal elastic deformations of supporting elements as well as the discharge arm vibration model, by making reasonable assumptions. The discharge arm system is simplified to single degree of freedom model, using the geometric invariant principle for three diagonal elastic deformation of supporting elements, and by the Laplace transform method gets the vibration model of the discharge arm system. To this, for reprint conveyor performance provides effective basis for smooth work.
\end{abstract}

\section{Keywords-materials; vibration; transport}

\section{INTRODUCTION}

End conveyor used in open-pit mine, is supported by three diagonal elastic supporting elements and the discharge arm, which is composed by lattice truss basic body. Belongs to ultral twice statically inclined support extended end lattice column [1], in its upper installation of belt conveyor, can realize the function of reproduced transport. As shown in figure 1 . When the materials have a certain kinetic energy in the discharge arm, cause its vibration, increases the dynamic load of the components; To highlight the dynamic load characteristics, its simplified into single degree of freedom vibration model [2].

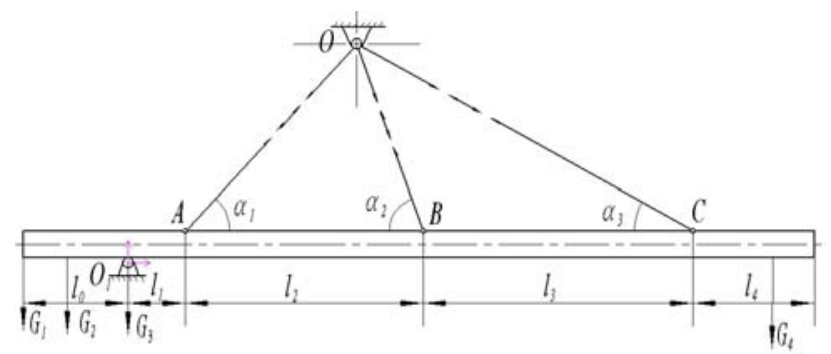

FIGURE I. FIGURE I. CONVEYOR MECHANISMS DIAGRAM

\section{ELONGATION FOR ELASTIC ELEMENT}

The assumptions for calculating the elongation of elastic element: body;

1) Discharge arm is regarded as a homogeneous rigid

\section{Z.B. $\mathrm{Li}$}

NHI Design and Research Institute, Shenyang, Liaoning, China

2) Material as a uniform distribution on the discharge arm, material quality $\mathrm{m} 01=390 \mathrm{~kg} / \mathrm{m}$, the quality of the conveyor belt $\mathrm{m} 02=190 \mathrm{~kg} / \mathrm{m}$.

3) Discharge arm as rigid body, A point to boundary, such as controlled beam, the moment of inertia J1, J2. Under the influence of disturbing force, the vibration of the discharge arm Angle is $\theta$.

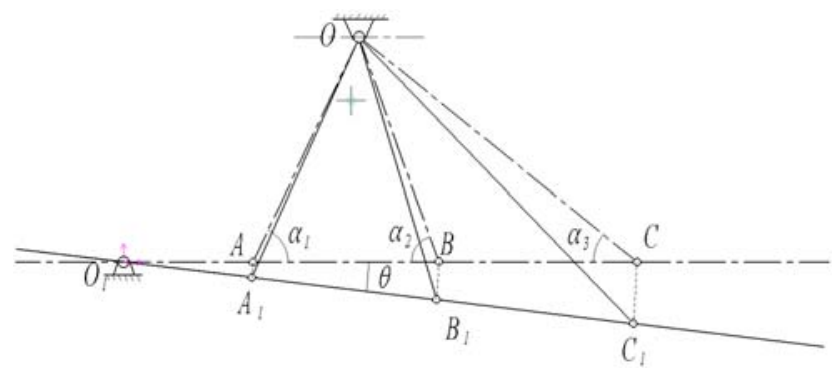

FIGURE II. ELASTIC DEFORMATION OF CONVEYOR MECHANISMS

Three elastic: $\mathrm{OA}, \mathrm{OB}, \mathrm{OC}$, elongation, respectively, to $\mathrm{OA}_{1}, \mathrm{OB}_{1}, \mathrm{OC}_{1}$. the deformation is shown in figure 2 .

According to the principle of geometric constant, obtains its elongation, respectively

$$
\angle O_{1} A A_{1}=\theta \text {, in } \triangle O A A_{1}
$$

$\beta_{1}=\angle O A A_{1}=\alpha_{1}+180^{\circ}-\angle O_{1} A A_{1}=\alpha_{1}+180^{\circ}-\left(180^{\circ}-\theta\right) / 2=90^{\circ}+\alpha_{1}+\theta / 2$ According to the law of cosines, there is

$$
\begin{gathered}
l_{O A 1}^{2}=l_{O A}^{2}+\left(l_{1} \theta\right)^{2}-2 l_{O A} \cdot\left(l_{1} \theta\right) \cos \beta_{1} \\
l_{O A 1}=\sqrt{l_{O A}^{2}+\left(l_{1} \theta\right)^{2}+2 l_{O A} \cdot\left(l_{1} \theta\right) \sin \left(\alpha_{1}+0.5 \theta\right)} \\
\square l_{O A}=l_{O A 1}-l_{O A}=l_{O A}\left(\sqrt{1+\frac{\left(l_{1} \theta\right)^{2}+2 l_{O A} \cdot\left(l_{1} \theta\right) \sin \left(\alpha_{1}+0.5 \theta\right)}{l_{O A}^{2}}}-1\right)
\end{gathered}
$$

For, $l_{O A}=19.76 \mathrm{~m} \approx 4 l_{1}=4 \times 5 \mathrm{~m}$

$$
\therefore x=\frac{\left(l_{1} \theta\right)^{2}+2 l_{O A} \cdot\left(l_{1} \theta\right) \sin \left(\alpha_{1}+0.5 \theta\right)}{l_{O A}^{2}} \leq|1|
$$




$$
\begin{aligned}
\square l_{O A} & =l_{O A 1}-l_{O A}=l_{O A}\left(1+\frac{1}{2} \frac{\left(l_{1} \theta\right)^{2}+2 l_{O A} \cdot\left(l_{1} \theta\right) \sin \left(\alpha_{1}+0.5 \theta\right)}{l_{O A}^{2}}-1\right) \\
& \approx l_{1} \cdot \sin \alpha_{1} \cdot \theta
\end{aligned}
$$

By the same token, then obtained,

$$
\begin{gathered}
\beta_{2}=\angle O B B_{1}=\alpha_{2}+\angle O_{1} B B_{1}=\alpha_{2}+\left(180^{\circ}-\theta\right) / 2=90^{\circ}+\alpha_{2}-\theta / 2 \\
l_{1}+l_{2}=5+18.36=23.4 m, \quad l_{O B}=14.86 \approx 15 m \\
\square l_{O B}=l_{O B 1}-l_{O B}=l_{O B}\left(1+\frac{1}{2} \frac{\left(\left(l_{1}+l_{2}\right) \theta\right)^{2}+2 l_{O B} \cdot\left(\left(l_{1}+l_{2}\right) \theta\right) \sin \left(\alpha_{2}-0.5 \theta\right)}{l_{O B}^{2}}-1\right) \\
\approx\left(l_{1}+l_{2}\right) \cdot \sin \alpha_{2} \cdot \theta \quad \text { Make } \\
l_{1}+l_{2}+l_{3}=5+18.36+21.6=45 m, \quad l_{O C}=30.32 m, \alpha_{3}=27^{\circ}, \\
x=\sqrt{1+\frac{\left(\left(l_{1}+l_{2}+l_{3}\right) \theta\right)^{2}+2 l_{O C} \cdot\left(\left(l_{1}+l_{2}+l_{3}\right) \theta\right) \sin \left(\alpha_{3}-0.5 \theta\right)}{l_{O C}^{2}}},
\end{gathered}
$$

Get $\theta \max =0.5^{\circ}$, and the relevant data into the expression of $\mathrm{x}, \mathrm{x}=0.135<1$, the series expansion are available, and namely,

$$
\begin{aligned}
\square l_{O C} & =l_{O C 1}-l_{O C}=l_{O C}\left(1+\frac{1}{2} \frac{\left(\left(l_{1}+l_{2}+l_{3}\right) \theta\right)^{2}+2 l_{O C} \cdot\left(\left(l_{1}+l_{2}+l_{3}\right) \theta\right) \sin \left(\alpha_{3}-0.5 \theta\right)}{l_{O C}^{2}}-1\right) \\
& \approx\left(l_{1}+l_{2}+l_{3}\right) \cdot \sin \alpha \cdot \theta
\end{aligned}
$$

A. Discharge Arm and The Discharging Arm Material

1) The kinetic equation of discharge arm

$$
\begin{aligned}
T_{1} & =\frac{1}{2} J_{1} \dot{\theta}^{2}+\frac{1}{2} J_{2} \dot{\theta}^{2} \\
J_{1} & =\frac{1}{3} m_{1} l_{0}^{2} \\
m_{1} & =\frac{m \cdot l_{0}}{l_{0}+l_{1}+l_{2}+l_{3}+l_{4}} \\
J_{2} & =\frac{1}{3} m_{2}\left(l_{1}+l_{2}+l_{3}+l_{4}\right)^{2} \\
m_{2} & =\frac{m}{l_{0}+l_{1}+l_{2}+l_{3}+l_{4}} \cdot\left(l_{1}+l_{2}+l_{3}+l_{4}\right)
\end{aligned}
$$

The kinetic energy equation of concentration quality body on the discharge arm

$$
T_{2}=\frac{1}{2} \frac{G_{1}}{g}\left(\dot{\theta} l_{0}\right)^{2}+\frac{1}{2} \frac{G_{2}}{g}\left(\dot{\theta} l_{5}\right)^{2}
$$

The kinetic energy equation of the material on the discharge arm

$$
\begin{aligned}
& T_{3}=\frac{1}{2}\left(J_{3}+J_{4}\right) \dot{\theta}^{2} \\
& J_{3}=\frac{1}{3} m_{3} l_{0}^{2} \\
& m_{3}=m_{01} l_{0}(\mathrm{~kg}) \\
& J_{4}=\frac{1}{3} m_{4}\left(l_{1}+l_{2}+l_{3}+l_{4}\right)^{2}
\end{aligned}
$$

$$
m_{4}=m_{01}\left(l_{1}+l_{2}+l_{3}+l_{4}\right) \quad(\mathrm{kg})
$$

The kinetic equations of the conveyor belt on the discharge arm

$$
\begin{aligned}
& T_{4}=\frac{1}{2}\left(J_{5}+J_{6}\right) \dot{\theta}^{2} \\
& J_{5}=\frac{1}{3} m_{5} l_{0}^{2} \\
& m_{5}=m_{02} l_{0}(\mathrm{~kg}) \\
& J_{6}=\frac{1}{3} m_{6}\left(l_{1}+l_{2}+l_{3}+l_{4}\right)^{2} \\
& m_{6}=m_{02}\left(l_{1}+l_{2}+l_{3}+l_{4}\right)(\mathrm{kg}) \\
& m=m_{1}+m_{2}+m_{3}+m_{4}+m_{5}+m_{6}
\end{aligned}
$$

\section{B. The Potential Energy of The Discharge Arm}

Take the equilibrium position of discharge arm to position of zero potential energy in the system, the discharge arm in tiny Angle theta $(\theta)$ place. Then, potential energy changes, which refers system relative to the amount of in position of zero potential energy[3],

$$
\begin{gathered}
V=\frac{1}{2} k_{1}\left(\theta l_{1}\right)^{2}+\frac{1}{2} k_{2}\left(\theta\left(l_{1}+l_{2}\right)\right)^{2}+\frac{1}{2} k_{3}\left(\theta\left(l_{1}+l_{2}+l_{3}\right)\right)^{2} \\
k_{1}=k_{O A}=\frac{E_{O A} F}{l_{O A}} \\
k_{2}=k_{O B}=\frac{E_{O B} F_{O B}}{l_{O B}} \\
k_{3}=k_{O C}=\frac{E_{O C} F_{O C}}{l_{O C}} \\
J_{7}=\frac{G_{1}}{g} l_{0}^{2}+\frac{G_{2}}{g} l_{5}^{2}
\end{gathered}
$$

Plug into the Laplace equation,

$$
\begin{gathered}
\frac{d}{d t}\left(\frac{\partial T}{\partial \dot{\theta}}\right)-\frac{\partial T}{\partial \theta}+\frac{\partial V}{\partial \theta}=0 \\
\frac{d}{d t}\left(\frac{\partial T}{\partial \dot{\theta}}\right)=\left(J_{1}+J_{2}+J_{3}+J_{4}+J_{5}+J_{6}+\frac{G_{1}}{g} l_{0}^{2}+\frac{G_{2}}{g} l_{5}^{2}\right) \ddot{\theta} \\
\frac{\partial V}{\partial \theta}=\left(k_{1} l_{1}^{2}+k_{2}\left(l_{1}+l_{2}\right)^{2}+k_{3}\left(l_{1}+l_{2}+l_{3}\right)^{2}\right) \theta
\end{gathered}
$$

Vibration equation for,

From it,

$$
J \dot{\theta}+K \theta=0
$$

$$
\begin{aligned}
& J=\left(J_{1}+J_{2}+J_{3}+J_{4}+J_{5}+J_{6}+\frac{G_{1}}{g} l_{0}^{2}+\frac{G_{2}}{g} l_{5}^{2}\right) \\
& K=\left(k_{1} l_{1}^{2}+k_{2}\left(l_{1}+l_{2}\right)^{2}+k_{3}\left(l_{1}+l_{2}+l_{3}\right)^{2}\right)
\end{aligned}
$$


Make $\omega_{0}^{2}=K / J$,

The form of differential equations, $\theta=e^{r t}$, and its solutions

$$
\begin{gathered}
\theta=C_{1} \cos \omega_{0} t+C_{2} \sin \omega_{0} t \\
A=\sqrt{C_{1}^{2}+C_{2}^{2}}, \tan \alpha=C_{1} / C_{2} \\
\theta=A \sin \left(\omega_{0} t+\alpha\right)
\end{gathered}
$$

Natural frequency,

$$
\omega_{0}=\sqrt{K / J}
$$

The

conditions, 1$) t=0, \theta=0 ; 2) t=0, \dot{\theta}=\dot{\theta}_{0}$.

By the initial conditions 1 ), there is $\alpha=0$, By the initial conditions 2), there is:

$$
A=\dot{\theta}_{0} / \omega_{0} .
$$

Vibration equation for,

$$
\theta=\frac{\dot{\theta}_{0}}{\omega_{0}} \sin \omega_{0} t
$$

\section{EXAMPLE CALCULATION}

The basic parameters of the conveyor:

The Weight of the discharge arm $m=38362 \mathrm{~kg}$, the material Weight $\mathrm{m} 01=390 \mathrm{~kg} / \mathrm{m}$, the Weight of the belt $\mathrm{m} 02=190 \mathrm{~kg} / \mathrm{m}$;

$G_{1}=35000 \mathrm{~N}, G_{2}=140000 \mathrm{~N}, G_{3}=80000 \mathrm{~N}, G_{4}=53000 \mathrm{~N}$, take $\theta=0.5^{\circ}, t_{1}=3 \mathrm{~s}, \mathrm{t}>\mathrm{t}_{1}=3 \mathrm{~s}$

$$
\begin{gathered}
\alpha_{1}=46^{\circ}, \quad \alpha_{2}=70^{\circ}, \alpha_{3}=27^{\circ} ; \\
l_{0}=5 \mathrm{~m}, l_{1}=5 \mathrm{~m}, l_{2}=18.36 \mathrm{~m}, l_{3}=21.6 \mathrm{~m}, l_{4}=6.6 \mathrm{~m},
\end{gathered}
$$

$15=2.55 \mathrm{~m}, 16=5 \mathrm{~m}$;

$$
l_{O A}=19.76 m, l_{O B}=14.86 m, l_{O C}=30.32 m ;
$$

By formula (1) can be obtained:

$$
\square l_{O A}=l_{1} \cdot \sin \alpha_{1} \cdot \theta=5 \sin 46^{\circ} \cdot \frac{0.5^{\circ}}{57.3}=0.031385 \mathrm{~m}
$$

\begin{tabular}{|c|c|c|c|c|c|}
\hline Name & $\begin{array}{c}\text { Nume } \\
\text { rical }\end{array}$ & $\begin{array}{c}\text { Formul } \\
\text { a } \\
\text { numbe } \\
r\end{array}$ & Name & Numerical & $\begin{array}{c}\text { Formu } \\
\text { la } \\
\text { numbe } \\
\mathrm{r}\end{array}$ \\
\hline $\begin{array}{c}m_{1} \\
(\mathrm{~kg})\end{array}$ & $\begin{array}{c}3391 . \\
3\end{array}$ & (4) & $\begin{array}{c}J_{4} \\
\mathrm{kgm}^{2}\end{array}$ & $1.782 \times 10^{7}$ & (11) \\
\hline $\begin{array}{c}m_{2} \\
(\mathrm{~kg})\end{array}$ & 34971 & (6) & $\begin{array}{c}J_{5} \\
\mathrm{kgm}^{2}\end{array}$ & $7.92 \times 10^{3}$ & (14) \\
\hline $\begin{array}{c}m_{3} \\
(\mathrm{~kg})\end{array}$ & 1950 & (10) & $\begin{array}{c}J_{6} \\
\mathrm{kgm}^{2}\end{array}$ & $8.7 \times 10^{6}$ & (16) \\
\hline $\begin{array}{c}m_{4} \\
(\mathrm{~kg})\end{array}$ & $\begin{array}{c}20108 \\
.4\end{array}$ & (12) & $\begin{array}{c}J_{7} \\
\mathrm{kgm}^{2}\end{array}$ & $\begin{array}{c}1.78535 \times 1 \\
0^{5}\end{array}$ & (22) \\
\hline $\begin{array}{c}m_{5} \\
(\mathrm{~kg})\end{array}$ & 950 & (15) & $\underset{\mathrm{kgm}^{2}}{J}$ & $\begin{array}{c}5.7751 \times 10 \\
7\end{array}$ & (24) \\
\hline $\begin{array}{c}m_{6} \\
(\mathrm{~kg})\end{array}$ & $\begin{array}{c}9796 . \\
4\end{array}$ & (17) & $\begin{array}{c}k_{1} \\
\mathrm{KN} / \mathrm{m}\end{array}$ & $3.44 \times 10^{5}$ & (19) \\
\hline $\begin{array}{c}m \\
(\mathrm{~kg})\end{array}$ & $\begin{array}{c}71167 . \\
1\end{array}$ & (18) & $\begin{array}{c}k_{2} \\
\mathrm{KN} / \mathrm{m}\end{array}$ & $4.344 \times 10^{4}$ & (20) \\
\hline $\begin{array}{c}J_{1} \\
\mathrm{kgm}^{2}\end{array}$ & $\begin{array}{l}2.826 \\
1 \times 10^{4}\end{array}$ & (3) & $\begin{array}{c}k_{3} \\
\mathrm{KN} / \mathrm{m}\end{array}$ & $2.129 \times 10^{4}$ & (21) \\
\hline $\begin{array}{c}J_{2} \\
k g m^{2}\end{array}$ & $\begin{array}{c}3.1 \times 1 \\
0^{7}\end{array}$ & (5) & $\begin{array}{c}K \\
\mathrm{KN} / \mathrm{m}\end{array}$ & $6.76 \times 10^{7}$ & (26) \\
\hline $\begin{array}{c}J_{3} \\
\mathrm{kgm}^{2}\end{array}$ & $\begin{array}{l}1.625 \\
\times 10^{4}\end{array}$ & (9) & $\begin{array}{c}\omega_{0} \\
\mathrm{rad} / \mathrm{s}\end{array}$ & 1.082 & (27) \\
\hline
\end{tabular}

According to the geometric constant principle can be obtained:

$$
\square l_{O B}=0.1469 \mathrm{~m}, \square l_{O C}=0.2825 \mathrm{~m}
$$

By formula (3), (4), (19) can be obtained:

$$
J_{1}=\frac{1}{3} m_{1} l_{0}^{2}=\frac{1}{3} \times 3391.3 \times 5^{2} \approx 2.8261 \times 10^{4} \mathrm{kgm}^{2}
$$

The other parameters of end conveyor calculation methods are same as above, as shown in table (1).
TABLEI.

CONVEYOR EXAMPLE CALCULATION PARAMETERS

By formula (24) shows that the vibration equation (29).of discharge arm system

$$
\theta=\frac{\dot{\theta}_{0}}{\omega_{0}} \sin \omega_{0} t=\frac{\dot{\theta}_{0}}{1.082} \sin 1.082 t
$$

The vibration equation of discharge arm system is shown as follows:

$$
\begin{gathered}
\theta=-4.76 \times 10^{-8} \sin 1.082 t+5.15 \times 10^{-8} t(\mathrm{rad}) \\
\text { IV. CONCLUSION }
\end{gathered}
$$

By the above discussion and calculation, the following conclusions can be concluded:

It is reasonable to simplify the discharge arm system as linear single degree of freedom vibration system in the process of the material fall on the discharge conveyor arm. The determination of the model assumptions and system of generalized degrees of freedom is consistent with the actual situation and the calculated results.

Discharge arm system is super quadratic statically indeterminate system, and using geometric invariant principle to calculate the three oblique elastic supporting elements of deformation. And the deformation results consistent with the actual mechanism and working conditions.

\section{ACKNOWLEDGEMENTS}

This work was financially supported by Chinese National 863 Project(2012AA062002). 


\section{REFERENCES}

[1] $\mathrm{Hu}$ Yandong, The elastic stability analysis of indeterminate truss structure in construction crane[D] Harbin Institute Of Technology. 2010.6 .

[2] He Xiaocong, The calculation method of the vibration of single degree of freedom system reliability[J]. Journal of Kunming University of Science and Technology, pp.50-52. 1998.4(23).

[3] Lin Guiyu, Li Zhaoyong, The Material Traces Determine and Significance of Reprinted Belt Conveyor [J]. International Conference on Mechanics and Civial Engineering, 2014. 